\title{
Creation of gap solitons in Bose-Einstein condensates
}

\author{
O. Zobay, E. M. Wright, and P. Meystre \\ Optical Sciences Center, University of Arizona, Tucson, Arizona 85721
}

\begin{abstract}
We discuss a method to launch gap soliton-like structures in atomic Bose-Einstein condensates confined in optical traps. Bright vector solitons consisting of a superposition of two hyperfine Zeeman sublevels can be created for both attractive and repulsive interactions between the atoms. Their formation relies on the dynamics of the atomic internal ground states in two far-off resonant counterpropagating $\sigma^{+}-\sigma^{-}$polarized laser beams which form the optical trap. Numerical simulations show that these solitons can be prepared from a one-component state provided with an initial velocity.
\end{abstract}

PACS numbers: 03.75.Fi, 05.30.Jp, 32.80.Pj

\section{INTRODUCTION}

The Gross-Pitaevskii equation (GPE) has been used successfully in the recent past to explain various experiments on atomic Bose-Einstein condensates (see, e.g., the references in 1,21), and its validity for the description of the condensate dynamics at zero temperature is now well accepted. A further confirmation would be provided by the observation of solitary matter waves, the existence of which is generic to nonlinear Schrödinger wave equations such as the GPE [3]. Such solitary waves could also find applications in the future, e.g., in the diffractionless transport of condensates.

Various theoretical studies of this problem have already been performed, predicting in particular the existence of bright solitons, with corresponding spatially localized atomic density profiles, for condensates with attractive interactions [2]. Research on condensates with repulsive interactions has focused on the formation of gray solitons which correspond to dips in the atomic density. Their creation was investigated in Refs. [1, 1 , their general properties were discussed in [5], and Ref. [2] worked out their analogy to the Josephson effect.

Complementary and previous to this work, the formation of atomic solitons was also examined theoretically in the context of nonlinear atom optics [6 11]. In these studies the interaction between the atoms was assumed to result from laser-induced dipole-dipole forces, but this theory has not been experimentally tested so far.

The reliance on attractive interactions to achieve bright matter-wave solitons in Bose-Einstein condensates is of course a serious limitation, due to the difficulties associated with achieving condensation in the first place for such interactions. The purpose of the present article is the theoretical exposition of an experimentally realizable geometry that allows one to create bright gap soliton-like structures in Bose condensates, for both attractive and repulsive signs of the two-body scattering length. Gap solitons result from the balance of nonlinearity and the effective linear dispersion of a coupled system, e.g., counterpropagating waves in a grating structure, and appear in the gaps associated with avoided crossings. Gap solitons have previously been studied in a variety of physical contexts, but particularly in nonlinear optics [12]. They were also studied in the framework of nonlinear atom optics [7], but in this case the two states involved are connected by an optical transition, and the effects of spontaneous emission can cause significant problems [8].

Several main reasons motivate our renewed interest in this problem. First, we already mentioned that bright gap solitons are known to exist in nonlinear systems irrespective of whether the nonlinear interaction is repulsive or attractive [13]. With regard to atomic condensates this means that they should be observable, at least in principle, also for $\mathrm{Na}$ and $\mathrm{Rb}$ where the positive interatomic scattering length gives rise to a repulsive mean interaction. Further, the study of bright solitary waves is of interest as they might be easier to detect than gray ones, and they could find future applications, e.g. in atomic interferometry [11]. An additional reason to study atomic gap solitons is the fact that they consist inherently of a superposition of two internal states, in our case two different Zeeman sublevels of the atomic ground state. As such, they offer a further example of a multicomponent Bose condensate the study of which has already received much interest recently 14 16. Finally, the recent demonstration of far-off resonant dipole traps for condensates opens up the way to the "easy" generation and manipulation of such spinor systems.

This paper is organized as follows. Section II describes our model. The physics relevant for the generation of gap solitons as well as orders of magnitudes for the various experimental parameters involved are discussed in Sec. III, while Sec. IV presents a summary of our numerical results. Finally, conclusions are given in Sec. V.

\section{THE MODEL}

The situation we consider for the generation of atomic gap solitons makes use of the recently achieved confinement of Bose condensates in far off-resonant optical dipole traps [17. We consider explicitly a trap consisting of two focused laser beams of frequency $\omega_{l}$ counterpropagating in the $Z$-direction and with polarizations $\sigma^{+}$ and $\sigma^{-}$, respectively. These lasers are used to confine a Bose condensate which is assumed for concreteness to 
consist of $\mathrm{Na}$ atoms. The condensate is initially prepared in the $\left|g, F_{g}=1, M_{g}=-1\right\rangle$ atomic ground state. For lasers far detuned from the resonance frequency $\omega_{a}$ of the nearest transition to an excited hyperfine multiplet $\left|e, F_{e}, M_{e}=-F_{e}, \ldots, F_{e}\right\rangle$ the dynamics of a single atom in the trap can be described by an effective Hamiltonian of the form 18

$$
\begin{aligned}
H_{e f f}= & \frac{\mathbf{P}^{2}}{2 m}+d_{0} \hbar \delta^{\prime}(\mathbf{R})|0\rangle\langle 0| \\
& +d_{1} \hbar \delta^{\prime}(\mathbf{R})(|-1\rangle\langle-1|+| 1\rangle\langle 1|) \\
& +d_{2} \hbar \delta^{\prime}(\mathbf{R})\left(|1\rangle\left\langle-1\left|e^{2 i K_{l} Z}+\right|-1\right\rangle\langle 1| e^{-2 i K_{l} Z}\right),
\end{aligned}
$$

which is derived by adiabatically eliminating the excited states in the dipole and rotating wave approximations. In the Hamiltonian (1), the operators $\mathbf{R}$ and $\mathbf{P}$ denote the center-of-mass position and momentum of the atom of mass $m$, the ket $|j\rangle$ labels the magnetic sublevel of the $\mathrm{Na}$ ground states, $|j\rangle \leftrightarrow\left|g, F_{g}=1, M_{g}=j\right\rangle$, and $K_{l}=\omega_{l} / c$. Furthermore,

$$
\delta^{\prime}(\mathbf{R})=\delta s(\mathbf{R}) / 2,
$$

where we have introduced the detuning $\delta=\omega_{l}-\omega_{a}$ and the position-dependent saturation parameter

$$
s(\mathbf{R})=\frac{\mathcal{D}^{2} \mathcal{E}^{2}(\mathbf{R})}{\delta^{2}+\Gamma^{2} / 4} \simeq \frac{\mathcal{D}^{2} \mathcal{E}^{2}(\mathbf{R})}{\delta^{2}} .
$$

In this expression, $\mathcal{D}$ denotes the reduced dipole moment between the states $|g\rangle$ and $|e\rangle, \Gamma$ is the upper to lower state spontaneous emission rate, and $\mathcal{E}(\mathbf{R})$ is the slowly varying laser field amplitude at point $\mathbf{R}$, the plane-wave factors $\exp \left[i\left( \pm K_{l} Z-\omega_{l} t\right)\right]$ having already been removed from the counterpropagating waves. In the following, we assume that $\mathcal{E}$, which is identical for both fields, varies only in the transverse $X$ - and $Y$-directions and is constant along the trap axis $Z$ : This approximation is valid if the longitudinal extension of the confined BEC is much less than the Rayleigh range of the trapping fields, a condition we assume is satisfied. The numerical coefficients $d_{j}$, which depend on the specific value of $F_{e}$, are of the order of or somewhat less than unity. Note that except insofar as $\Gamma$ appears in the saturation parameter $s(\mathbf{R})$, the effects of spontaneous emission are neglected in this description!.

\footnotetext{
${ }^{1}$ In the discussion of the atomic dynamics and Eq. (1) we have assumed that the initial state is coupled only to one excited hyperfine multiplet. However, in the optical trap the detuning of the laser frequency is large compared even to the fine structure splitting of the excited states, so that in principle several different hyperfine multiplets should be taken into account. Fortunately, the coupling to any of these multiplets gives rise to addititional contributions to Eq. (位) which are of the same analytical structure as the one given above. Only
}

The first term in the single-particle Hamiltonian (11) describes the quantized center-of-mass atomic motion, the second and third terms the (position dependent) light-shifts of the $j=0, \pm 1$ states, and the final term, proportional to $d_{2}$, describes the coupling between the $j= \pm 1$ states by the counterpropagating fields. For example, coupling between the $j=-1$ and $j=1$ states arises from the process involving absorption of one $\sigma^{+}$ photon and subsequent re-emission of a $\sigma^{-}$photon. However, since the circularly polarized fields are counterpropagating this process also involves a transfer of linear momentum $2 \hbar K_{l}$ along the $Z$-axis, and this accounts for the appearance of the spatially periodic factors, or gratings, $\exp \left( \pm 2 i K_{l} Z\right)$ in the coupling terms. As shown below, these gratings provide the effective linear dispersion which allows for gap solitons in combination with the nonlinearity due to many-body effects.

To describe the dynamics of the Bose condensate we introduce the macroscopic wave function $\boldsymbol{\Psi}(\mathbf{R}, t)=$ $\left[\Psi_{1}(\mathbf{R}, t), \Psi_{-1}(\mathbf{R}, t)\right]^{T}$ normalized to the total number of particles $N$. Here $\Psi_{0}$ is omitted as it is coupled to $\Psi_{ \pm 1}$ neither by $H_{\text {eff }}$ nor by the nonlinearity if it vanishes initially, which we assume in the following. The time evolution of the spinor $\boldsymbol{\Psi}(\mathbf{R}, t)$ is determined by the twocomponent Gross-Pitaevskii equation

$$
\begin{aligned}
i \hbar \frac{\partial \boldsymbol{\Psi}}{\partial t} & =H_{e f f} \mathbf{\Psi}(\mathbf{R}, t) \\
& +\left(\begin{array}{c}
{\left[U_{a}\left|\psi_{1}(\mathbf{R}, t)\right|^{2}+U_{b}\left|\psi_{-1}(\mathbf{R}, t)\right|^{2}\right] \psi_{1}(\mathbf{R}, t)} \\
{\left[U_{b}\left|\psi_{1}(\mathbf{R}, t)\right|^{2}+U_{a}\left|\psi_{-1}(\mathbf{R}, t)\right|^{2}\right] \psi_{-1}(\mathbf{R}, t)}
\end{array}\right) .
\end{aligned}
$$

In the following we approximate the nonlinearity coefficients by $U_{a} \approx U_{b}=U=4 \pi \hbar^{2} a_{s c} / m$ with $a_{s c}$ the $s$-wave scattering length.

To identify the key physical parameters for gap soliton formation, and to facilitate numerical simulations, it is convenient to re-express Eq. (4) in a dimensionless form by introducing scaled variables $\tau=t / t_{c}, \mathbf{r}=\mathbf{R} / l_{c}$ and $\psi_{j}=\Psi_{j} / \sqrt{\rho_{c}}$ with

$$
\begin{aligned}
t_{c} & =1 /\left(d_{2} \delta_{0}^{\prime}\right), \\
l_{c} & =t_{c} \cdot \hbar K / m, \\
\rho_{c} & =\left|d_{2} \hbar \delta_{0}^{\prime} / U\right|,
\end{aligned}
$$

where $\delta_{0}^{\prime}=\delta^{\prime}(\mathbf{R}=0)$. Note that for our choice of $d_{2}=$ $-1 / 4$, and for red detuning, we have $d_{2} \delta_{0}^{\prime}>0$. Equation (4) then reads

$$
i \frac{\partial \boldsymbol{\psi}}{\partial \tau}=\left[\begin{array}{cc}
-M \Delta+\frac{d_{1} \delta^{\prime}(\mathbf{r})}{d_{2} \delta_{0}^{\prime}} & e^{2 i k_{l} z} \delta^{\prime}(\mathbf{r}) / \delta_{0}^{\prime} \\
e^{-2 i k_{l} z} \delta^{\prime}(\mathbf{r}) / \delta_{0}^{\prime} & -M \Delta+\frac{d_{1} \delta^{\prime}(\mathbf{r})}{d_{2} \delta_{0}^{\prime}}
\end{array}\right]\left(\begin{array}{c}
\psi_{1} \\
\psi_{-1}
\end{array}\right)
$$

the values of $d_{j}, \mathcal{D}$, and $\Gamma$ are different. This means that Eq. (1) may still be used in this case, the effects of the additional multiplets being included as modifications of the values of the coefficients $d_{j}$. For simplicity, however, we will use the values of the $F_{g}=1 \rightarrow F_{e}=1$ transition in the following, i.e., $d_{0}=1 / 2, d_{1}=1 / 4$, and $d_{2}=-1 / 4$ [18]. 


$$
+\left[\operatorname{sgn}\left(d_{2} \hbar \delta_{0}^{\prime} / U\right)\left(\left|\psi_{1}\right|^{2}+\left|\psi_{-1}\right|^{2}\right)\right]\left(\begin{array}{c}
\psi_{1} \\
\psi_{-1}
\end{array}\right),
$$

where $\Delta$ is the Laplacian in scaled variables, and we have introduced the dimensionless mass-related parameter

$$
M=d_{2} \delta_{0}^{\prime} m /\left(2 \hbar K_{l}^{2}\right),
$$

so that $k_{l}=K_{l} l_{c}=1 /(2 M)$.

\section{GAP SOLITONS}

In this section we discuss the conditions under which Eqs. (8) yield gap soliton solutions. Rather than reproducing the explicit analytic forms of these solutions, which are readily available in the literature, here we introduce the reduced equations which yield gap solitons, and discuss the physics underlying their formation. Estimates for the orders of magnitude of various parameters characterizing atomic gap solitons are also given.

\section{A. Reduced soliton equations}

Two key approximations underly the appearance of gap solitons: First, we neglect all transverse variations of the electromagnetic and atomic fields, thereby reducing the problem to one spatial variable $z$. Furthermore, we can set $\delta^{\prime}(\mathbf{r}) / \delta_{0}^{\prime}=1$. Second we express the atomic fields in the form

$$
\psi_{ \pm 1}(z, t)=\exp \left\{i\left[ \pm k_{l} z-(1 /(4 M)-1) \tau\right]\right\} \phi_{ \pm 1}(z, t),
$$

and we assume that the atomic field envelopes $\psi_{ \pm 1}(z, t)$ vary slowly in space in comparison to the plane-wave factors that have been separated out, so that only first-order spatial derivatives of the field envelopes need be retained and only the spatial harmonics indicated included. Under these assumptions Eqs. (8) reduce to

$$
\begin{aligned}
& i\left(\frac{\partial}{\partial \tau} \pm 2 k_{l} \frac{\partial}{\partial z}\right)\left(\begin{array}{c}
\phi_{1} \\
\phi_{-1}
\end{array}\right)=\left(\begin{array}{ll}
0 & 1 \\
1 & 0
\end{array}\right)\left(\begin{array}{c}
\phi_{1} \\
\phi_{-1}
\end{array}\right) \\
& +\operatorname{sgn}\left(d_{2} \hbar \delta_{0}^{\prime} / U\right)\left(\left|\phi_{1}\right|^{2}+\left|\phi_{-1}\right|^{2}\right)\left(\begin{array}{c}
\phi_{1} \\
\phi_{-1}
\end{array}\right) .
\end{aligned}
$$

Aceves and Wabnitz 13 have shown that these dimensionless equations have explicit travelling solitary wave solutions of hyperbolic secant form. Thus, the optical trapping geometry we propose here can support atomic gap solitons under the appropriate conditions.

Having established that our system can support gap solitons our goal in the remainder of this paper is to demonstrate through numerical simulations that these solitons, or at least a remnant of them, can arise for realistic atomic properties and that they can be created from physically reasonable initial conditions. In particular, the exact gap solitons solutions are coherent superpositions of the $j= \pm 1$ states where the phase and amplitude of the superposition varies spatially in a specific manner: it is not a priori clear that these gap solitons can be accessed from an initial state purely in the $j=-1$ state for example. Furthermore, inclusion of transverse variations and spatial derivatives beyond the slowly-varying envelope approximation introduced above could, in principle, destroy the solitons 19]. For the numerical simulations to be presented here we work directly with Eqs. (8) which does not invoke these approximations.

\section{B. Intuitive soliton picture}

A simple and intuitively appealing explanation of the reason why Eq. (8) supports soliton solutions goes as follows: Consider first the one-dimensional nonlinear Schrödinger equation

$$
i \dot{\psi}=-M \partial^{2} \psi / \partial z^{2}+g|\psi|^{2} \psi .
$$

This equation has bright soliton solutions if the effects of dispersion and nonlinearity can cancel each other. For this to happen, it is necessary that $M g<0$. In the usual case the mass-related coefficient $M$ is positive, so that bright solitons can only exist in condensates with attractive interactions $g<0$. But consider now the dispersion relation $\omega(k)=M k^{2} \pm \sqrt{1+k^{2}}$ for the linear part of Eq. (8) obtained after neglecting the transverse dimensions and performing the transformation

$$
\psi_{ \pm 1}=a_{ \pm 1} \exp \left\{i\left[k_{ \pm 1} z-\omega(k) \tau\right]\right\} .
$$

Thereby, $k_{ \pm 1}=k \pm k_{l}$ for the $j= \pm 1$ states, and $k$ is a relative longitudinal wave vector. The dispersion relation consists of two branches, which in the absence of linear coupling take the form of two parabolas corresponding to the free dynamics of the internal states $| \pm 1\rangle$. However, the linear coupling between these states results in an avoided crossing at $k=0$, see Fig. 1. If the system is in a superposition of eigenstates pertaining to the lower branch of the dispersion relation, then at the crossing it can be ascribed a negative effective mass. One can thus expect that in this case the system can support soliton solutions even though the interaction is repulsive. For an attractive interaction, in contrast, soliton creation should be possible in all regions of the spectrum with positive effective mass.

From the dispersion relation picture, one can easily infer further properties of repulsive interaction solitons. First, they will only exist for weak enough dispersion, as the lower branch of the dispersion curve has a region with negative curvature only as long as the dimensionless mass $M<0.5$. Also, the maximum possible velocity can be estimated to be of the order of $\left[1-(2 M)^{2 / 3}\right]^{3 / 2}$ which is the group velocity at the points $\pm k_{0}= \pm \sqrt{(2 M)^{-2 / 3}-1}$ of vanishing curvature in the dispersion relation. Finally, 
for a soliton at rest the contributions of the internal states $|1\rangle$ and $|-1\rangle$ will approximately be equal, but solitons traveling with increasing positive, resp. negative, velocity will be increasingly dominated by the $|1\rangle$, resp. $|-1\rangle$, contribution.

This qualitative discussion is in agreement with the analytic results of Ref. 13]. More precisely, the solutions of Ref. 13] are solitary waves. In the following, we will be concerned with the creation of long-lived localized wave packet structures which are brought about by the interplay between nonlinearity and dispersion described above. We will continue to refer to these structures as gap solitons for simplicity.

\section{Soliton estimates}

We now turn to a discussion of the typical orders of magnitude which characterize the soliton solutions of Eq. (4). We note from the outset that the analytical solutions of Ref. [13], as well as our numerical simulations, indicate that these characteristic scales can be directly inferred from the scale variables in Eqs. (5)-(7) which bring the Gross-Pitaevskii equations into dimensionless form. For example, the spatial extension of the scaled wave function $\boldsymbol{\psi}$, as well as the total norm $\int d v\left(\left|\psi_{1}\right|^{2}+\left|\psi_{-1}\right|^{2}\right)$ are of order unity. 2

In order to obtain estimates for the characteristic length, time and density introduced in Eqs. (5)-(7) we use the parameter values of the $\mathrm{Na}$ experiment of Ref. [17] as a guidance. For Sodium, $\Gamma=61 \mathrm{MHz}$, the saturation intensity $I_{s}=6.2 \mathrm{~mW} / \mathrm{cm}^{2}$ and the resonance wavelength $\lambda_{a}=589 \mathrm{~nm}$. Choosing the trap wavelength to be far red-detuned from this value, with $\lambda_{l}=985 \mathrm{~nm}$, and a maximum laser intensity $I \approx 1 \mathrm{~kW} / \mathrm{cm}^{2}$ one obtains a characteristic scale $t_{c}$ for the time evolution of the condensate of the order of $50 \mu \mathrm{s}$. The characteristic length is obtained by multiplication with the recoil velocity $v_{\text {rec }}=1.8 \mathrm{~cm} / \mathrm{s}$, which yields $l_{c} \approx 1-2 \mu \mathrm{m}$. This yields the dimensionless mass $M \approx 0.1$. Finally, the order of magnitude of the characteristic density $\rho_{c}=10^{14}$ $\mathrm{cm}^{-3}$, which means that a soliton typically contains of the order of $\rho_{c} l_{c}^{3} \approx 100-1000$ atoms. These estimates are confirmed by our numerical simulations, which show that the typical extension of a soliton is several $l_{c}$ in the $z$-direction, about one $l_{c}$ in the transverse direction and it contains about 1000 atoms.

Our numerical simulations show that the maximum dimensionless atomic density in a soliton is always of the order of $\rho_{m}=0.1$, which appears to produce the nonlinearity necessary to balance the effects of disper-

\footnotetext{
${ }^{2}$ Note that the precise values of $d_{1}$ and $d_{2}$ are only of relevance for the scaling between Eqs. (44) and (8). They do not influence the essential physics of the system.
}

sion. From this value, it is possible to obtain a first estimate of the transverse confinement of the condensate required in our two-dimensional model: We assume that the transverse spatial dependence of the atomic density can be modeled as the normalized ground state density $g(X)$ of the harmonic trap potential $\delta^{\prime}(X)=m \omega_{x}^{2} X^{2} / 2$ [5]. The soliton density can hence be roughly estimated as $\rho(\mathbf{R})=\Theta(Z) \Theta(\Delta Z-Z) g(X) N / \Delta Z$, where $\Theta(Z)$ is the Heaviside step function, $N$ a typical total number of atoms in the soliton and $\Delta Z$ its length. From this condition, and using the typical values for $N$ and $\Delta Z$ previoulsy discussed leads to a lower limit for $\omega_{x}$ in the range between 100 and $1000 \mathrm{~Hz}$. Altogether, these various estimates are well within experimental reach.

\section{NUMERICAL RESULTS}

Having characterized the idealized gap soliton solutions of Eq. (4) we now investigate whether they can be accessed from realistic initial conditions. To this end, we study numerically the following situation. A condensate of $N_{0}$ atoms in the internal state $|-1\rangle$ is initially prepared in a conventional optical dipole trap which provides only a tranverse confinement potential $V_{c}$. This potential is assumed to be Gaussian, with a trapping frequency $\omega_{x}$ at the bottom. Axially, the condensate is confined by a harmonic magnetic trap of frequency $\omega_{z}$. At $t=0$ the magnetic trap is turned off and the polarizations of the trapping light fields are switched to the $\sigma^{+}-\sigma^{-}$configuration, with $V_{c}$ being unchanged.

The simulations are performed in two spatial dimensions only, $x$ and $z$, as this can already be expected to capture the relevant physics without requiring excessive computational resources. We concentrate on the more interesting case of a condensate with repulsive interactions since that is the new case in which solitons are expected. In order to estimate atom numbers the transformation between Eqs. (8) and (4) is performed after replacing $U$ by $U / \sqrt{\left\langle X^{2}\right\rangle}$ where the variance $\left\langle X^{2}\right\rangle$ is determined from the two-dimensional wave packet structure in question.

The main purpose of the numerical simulations is to show that gap solitons can be formed out of condensate wave functions whose initial parameters lie within a relatively broad range. It is only necessary to choose $\omega_{x}$, $\omega_{z}$, and $N_{0}$ such that the spatial extension of the initial condensate and the atom number are comparable to typical soliton values. They need not take on precisely defined values and the initial wave function does not have to match closely the form of a soliton.

However, the condensate will not couple effectively to a soliton if it is at rest initially. Such a situation corresponds to the point with $k=k_{l}$ in Fig. 11 where the effective mass is still positive $\left(k_{l}>k_{0}\right)$. The key to an efficient generation of solitons is therefore to provide the condensate with an initial velocity $V_{i n}=\hbar K_{i n} / m$ close to the recoil velocity $V_{r}$ in the $Z$-direction. This may 
be achieved, e.g., by suddenly displacing the center of the magnetic trap. The initial wave function can then be written as $\psi_{-1}(x, z, 0)=\psi_{g}(x, z, 0) \exp \left(i k_{i n} z\right)$ with $\psi_{g}(x, z, 0)$ the ground state of the combined optical and magnetic trap and $k_{i n}=K_{i n} l_{c}$ [3]. It is thus placed in the vicinity of the avoided crossing. Experimentally, condensates have already been accelerated to velocities in this range by a similar method in connection with the excitation of dipole oscillations 20].

Figure 2 shows an illustrative example for the formation of a soliton out of the initial distribution. It depicts the evolution of the transverse averaged atomic density

$$
N(z ; \tau)=\int d x\left(\left|\psi_{1}(x, z ; \tau)\right|^{2}+\left|\psi_{-1}(x, z ; \tau)\right|^{2}\right),
$$

as a function of the scaled variables $z$ and $\tau$. In this example, $\lambda_{l}=985 \mathrm{~nm}$, the maximum intensity $I=0.88$ $\mathrm{kW} / \mathrm{cm}^{2}, \omega_{x}=6000 \mathrm{~s}^{-1}, \omega_{z}=5100 \mathrm{~s}^{-1}$, and $V_{i n}=$ $0.9 V_{r}$. The characteristic scales are thus $l_{c}=1.4 \mu \mathrm{m}$ and $t_{c}=77 \mu \mathrm{s}$, the coefficient $M=0.06$. We choose $\int d x d z\left|\psi_{-1}(x, z, 0)\right|^{2}=6.21$ which corresponds to an initial atom number of 2900, approximately. Figure 2 shows the formation of a soliton after an initial transient phase having a duration of $50 t_{c h a r}$, approximately. This transient phase is characterized by strong "radiation losses". They occur because half of the initial state pertains to the upper branch of the dispersion relation $\omega(k)$ which cannot sustain solitons. The shape of the created soliton is not stationary in time but appears to oscillate. Further examination shows that norm of the $j=-1$ state is slightly larger than that of the $j=1$ state, as is expected for a soliton moving slowly in negative $z$-direction [13]. Figure 3 shows the atomic density $P(x, z)=\left|\psi_{1}(x, z ; \tau)\right|^{2}+\left|\psi_{-1}(x, z ; \tau)\right|^{2}$ in the soliton at $\tau=100$. The soliton contains about 500 atoms. The inset depicts the longitudinally integrated density $\int d z\left(\left|\psi_{1}(x, z ; \tau)\right|^{2}+\left|\psi_{-1}(x, z ; \tau)\right|^{2}\right)$ and the transverse confinement potential in the shape of an inverted Gaussian. The soliton thus spreads out over half the width of the potential well, approximately.

Various numerical simulations were performed in order to assess the dependence of soliton formation on the various initial parameters. When changing the atom number $N_{0}$ a soliton was formed over the whole investigated range between 500 and 4000 atoms. With increasing $N_{0}$ and thus increasing effects of the nonlinearity the final velocity of the soliton changed from negative to positive values. For large $N_{0}$ a tendency to form two soliton wave packets out of the initial state was observed, however, the formation of each of these solitons is accompanied by large radiation losses which destabilize the other one. As to the transverse confinement parameter $\omega_{x}$ soliton formation was observed for $\omega_{x} \geq 4000 \mathrm{~s}^{-1}$. At $\omega_{x}=2000$ $\mathrm{s}^{-1}$ a stable structure was no longer attained which is in rough agreement with the estimate given above. Whereas these numbers indicate a relatively large freedom in the choice of $N_{0}$ and $\omega_{x}$ (for $\omega_{z}$ similar results can be expected) a somewhat more restrictive condition is placed on the initial velocity $V_{i n}$. Its value should be chosen from the interval between 0.8 and $1.0 V_{r}$ in order to guarantee soliton formation, the lower bound being determined by the point of vanishing curvature in the dispersion relation. For $V_{i n}>V_{r}$ the initial wave function is situated more and more on the upper branch of the dispersion relation so that the tendency to form solitons is diminished rapidly.

\section{SUMMARY AND CONCLUSION}

In conclusion, we have demonstrated that gap solitonlike structures can be created in a Bose condensate confined in an optical dipole trap formed by two counterpopagating $\sigma^{+}-\sigma^{-}$-polarized laser beams. Bright solitons can be formed not only for atomic species with attractive interactions but also in the repulsive case. This is rendered possible because the atoms can be ascribed a negative effective mass if their velocity is close to the recoil velocity. The repulsive interaction solitons are inherently superpositions of two hyperfine Zeeman sublevels. The discussion of characteristic scales and numerical simulations indicated that the actual observation of these structures should be achievable within the realm of current experimental possibilities.

In our theoretical treatment spontaneous emission was neglected, an approximation justified by the large detunings in the optical trap [17]. The effects of anti-resonant terms, which were also ignored, might be of more importance. This question, as well as three-dimensional numerical studies, could be the subject of future work.

\section{ACKNOWLEDGMENTS}

We have benefited from numerous discussions with $\mathrm{E}$. V. Goldstein. This work is supported in part by the U.S. Office of Naval Research Contract No. 14-91-J1205, by the National Science Foundation Grant PHY95-07639, by the U.S. Army Research Office and by the Joint Services Optics Program.

[1] T. F. Scott, R. J. Ballagh, and K. Burnett, Report No. cond-mat/9711111.

[2] W. P. Reinhardt and C. W. Clark, J. Phys. B 30, L785 (1997).

[3] S. A. Morgan, R. J. Ballagh, and K. Burnett, Phys. Rev. A 55, 4338 (1997).

[4] R. Dum, J. I. Cirac, M. Lewenstein, and P. Zoller, Report No. cond-mat/9710238.

[5] A. D. Jackson, G. M. Kavoulakis, and C. J. Pethick, Report No. cond-mat/9803116. 
[6] G. Lenz, P. Meystre, and E. W. Wright, Phys. Rev. Lett. 71, 3271 (1993).

[7] G. Lenz, P. Meystre, and E. W. Wright, Phys. Rev. A 50, 1681 (1994).

[8] K. J. Schernthanner, G. Lenz, and P. Meystre, Phys. Rev. A 50, 4170 (1994).

[9] W. Zhang, D. F. Walls, and B. C. Sanders, Phys. Rev. Lett. 72, 60 (1994).

[10] S. Dyrting, Weiping Zhang, and B. C. Sanders, Phys. Rev. A 56, 2051 (1997).

[11] M. Holzmann and J. Audretsch, Europhys. Lett. 40, 31 (1997).

[12] C. M. de Sterke and J. E. Sipe, in Progress in Optics, edited by E. Wolf (Elsevier, Amsterdam, 1994), Vol. XXXIII.

[13] A. B. Aceves and S. Wabnitz, Phys. Lett. A 141, 37 (1989).

[14] T.-L. Ho and V. B. Shenoy, Phys. Rev. Lett. 77, 2595 (1996).

[15] E. V. Goldstein and P. Meystre, Phys. Rev. A 55, 2935 (1997).

[16] C. J. Myatt, E. A. Burt, R. W. Ghrist, E. A. Cornell, and C. E. Wieman, Phys. Rev. Lett. 78, 586 (1997).

[17] D. M. Stamper-Kurn, M. R. Andrews, A. P. Chikkatur, S. Inouye, H.-J. Miesner, J. Stenger, and W. Ketterle, Phys. Rev. Lett. 80, 2027 (1998).

[18] C. Cohen-Tannoudji, in Fundamental Systems in Quantum Optics, edited by J. Dalibard, J.-M. Raimond, and J. Zinn-Justin (North-Holland, Amsterdam, 1992).

[19] A. R. Champneys, B. A. Malomed, and M. J. Friedman, Phys. Rev. Lett. 80, 4169 (1998).

[20] D. S. Durfee and W. Ketterle, Opt. Express 2, 299 (1998).

FIG. 1. Dispersion relation of the linear part of Eq. (8) for the parameter value $M=0.06$. The $k$-value associated with a condensate at rest is denoted by $k_{l}$, the point of vanishing curvature is indicated by $k_{0}$.

FIG. 2. Formation of a soliton out of an initial distribution. Depicted is the integrated density $N(z ; \tau)=\int d x\left(\left|\psi_{1}(x, z ; \tau)\right|^{2}+\left|\psi_{-1}(x, z ; \tau)\right|^{2}\right)$ in scaled variables. Parameter values given in the text.

FIG. 3. Atomic density $P(x, z)=\left|\psi_{1}(x, z ; \tau)\right|^{2}+\left|\psi_{-1}(x, z ; \tau)\right|^{2}$ of the soliton of Fig. 2 at $\tau=100$. The inset shows the integrated density $Q(x)=\int d z\left(\left|\psi_{1}(x, z ; \tau)\right|^{2}+\left|\psi_{-1}(x, z ; \tau)\right|^{2}\right)$ and the transverse confinement potential $V(x)$. 


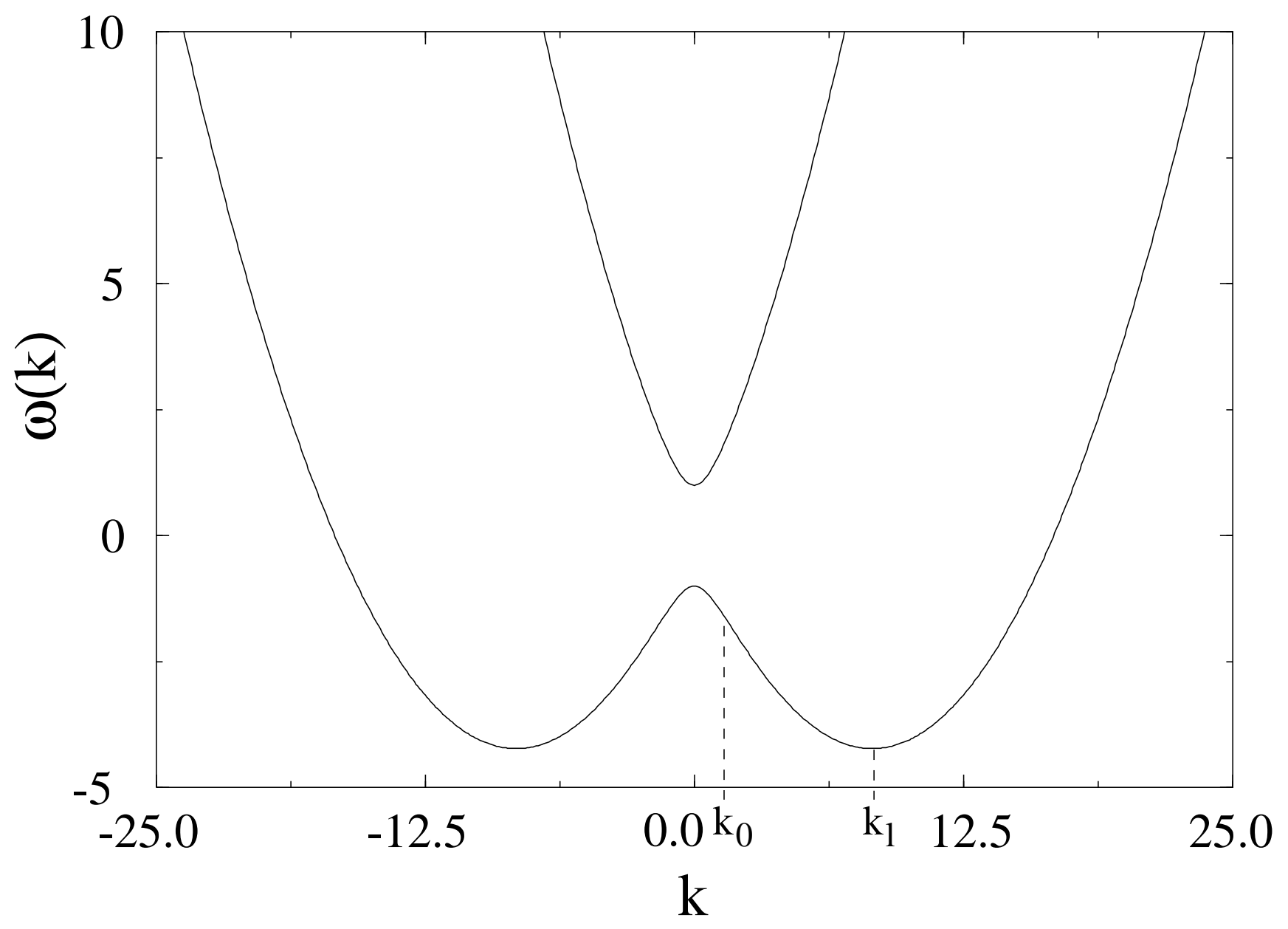


This figure "fig2.gif" is available in "gif" format from: http://arxiv.org/ps/cond-mat/9805228v1 
This figure "fig3.gif" is available in "gif" format from: http://arxiv.org/ps/cond-mat/9805228v1 